\title{
AMS FALL SECTIONAL SAMPLER
}

\section{Below, Elisenda Grigsby describes some invariants for braids, knots, and links, and connects their study to Fox's slice-ribbon conjecture, as an introduction to her Invited Address for the AMS Fall Eastern Sectional Meeting being held at the University of Delaware, September 29-30.}

J. Elisenda Grigsby

\section{Braids, Surfaces, and Homological Invariants}

I'm thrilled to be delivering an invited address at the fall eastern sectional meeting of the AMS. Rather than explaining the details of what I've worked on, I'll focus attention on why you should care.

For as long as mathematicians have studied knots and links-smooth embeddings of circles into $S^{3}$, considered up to isotopy - they have studied the smoothly embedded surfaces they bound. As with most of mathematics, one big reason they do it is that they can. Spend a few minutes doodling knot diagrams in your algebraic topology notebook (or watch Vi Hart's awesome "Snakes and Graphs" video on YouTube) and you'll see that every knot bounds a so-called checkerboard surface as in Figure 1. This surface is not guaranteed to be orientable, and typically isn't. Spend a bit more time doodling, though, and perhaps you'll rediscover Seifert's algorithm, which explicitly constructs an orientable surface in $S^{3}$ bounded by the knot.

Constructing surfaces bounded by a given knot is easy. It is much harder to determine whether any given surface one constructs has minimal possible complexity, as measured by its genus or, more generally, by the number of critical points of a Morse function inherited from an embedding.

Thanks to decades of (largely pre-YouTube) work, we know a lot more than we used to about questions of this

J. Elisenda Grigsby is professor of mathematics at Boston College and a deputy director at the Institute for Computational and EXperimental Research in Mathematics (ICERM) at Brown. Her email address is grigsbyj@bc. edu.

For permission to reprint this article, please contact: reprint-permission@ams.org.

DOI: http://dx.doi.org/10.1090/noti1710
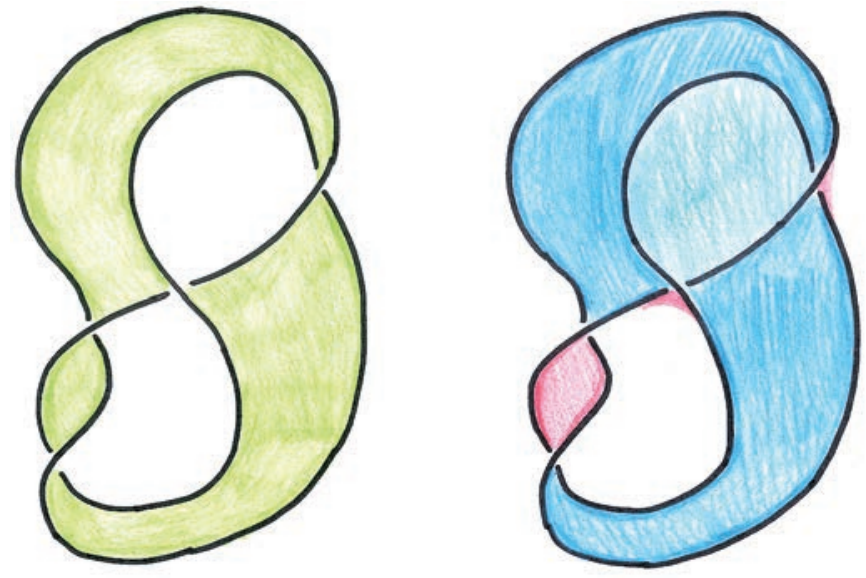

Figure 1. Every knot, such as the figure-eight knot pictured here, bounds a checkerboard surface and an orientable Seifert surface.

type. But there are a number of basic points on which we remain ignorant. I'll highlight just one.

Consider the standard radial function on $\mathrm{R}^{4}$. The fourball, $B^{4}$, is the set of points of radius at most 1 , and its boundary is the three-sphere, $S^{3}$. Over 50 years ago, Fox asked the following question: If a knot in $S^{3}$ bounds a smoothly embedded disk in $B^{4}$, does it bound a smoothly embedded disk in $B^{4}$ for which the radial function, when restricted to the embedding, has no local maxima?

The expectation (hope? dream?) that the answer to Fox's question is yes is known as the slice-ribbon conjecture, since knots satisfying the first condition are called slice, and knots satisfying the second are called ribbon. The question may seem easy to resolve. It isn't. In fact, it is wide open in every sense. Even mathematicians who have thought seriously about it would feel uncomfortable betting money on one side or the other.

Old nails call for new hammers. Some of the best modern tools we have for studying smoothly embedded surfaces in $B^{4}$ are homological invariants coming from 


\section{AMS FALL SECTIONAL SAMPLER}

quantum topology, gauge theory, and symplectic geometry. In brief, one begins with data specifying a knot (for example, a knot diagram) and constructs from this data an abstract chain complex whose homology is independent of the choices made in its construction. An embedded surface in $B^{4}$ induces a map on the complex, and this map is well behaved with respect to internal gradings in the theory. In particular, the Euler characteristic of the surface bounds the map's degree, and one uses this fact to obtain a lowerbound on the genus.

\section{Old nails call for new hammers.}

To illustrate the power of this idea and connect it back to Fox's slice-ribbon question, let me describe a classical family of examples from complex geometry, made famous by John Milnor. Let $p$ and $q$ be a pair of relatively prime positive integers, and consider the vanishing set of the polynomial $f(z, w)=z^{\mathrm{p}}+w^{\mathrm{q}}$ in $\mathbf{C}^{2}$. Intersect this vanishing set with a three-sphere centered at the origin, and the resulting knot sits as a slope $p / q$ curve on a standardly embedded torus. One natural way to construct a Seifert surface for this knot is by realizing it as the closure of a positive braid on $q$ strands and forming a surface compatible with this presentation. A glance at Figure 2 tells us that the oriented surface constructed in this way has Euler characteristic $p-q(p-1)$. Noting that its boundary is connected, we compute that its genus is $(p-1)(q-1) / 2$.

Milnor conjectured that one cannot find a lower genus surface bounded by the $(p, q)$ torus knot, even when the surface is allowed to lie in the interior of $B^{4}$. Milnor's conjecture was eventually proved in the 1990s by Kronheimer and Mrowka using gauge theory. It has since been reproved using many of the more recently defined homological invariants. Indeed, the work of Kronheimer-Mrowka, com-

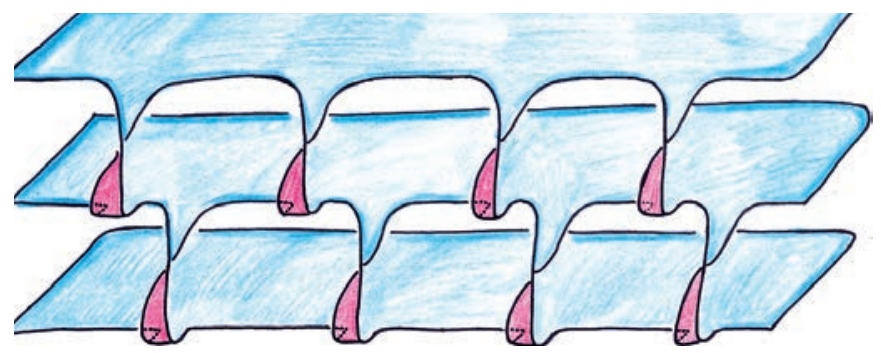

Figure 2 . This braided surface bounded by a $(3,4)$-torus knot has genus $(3-1)(4-1) / 2=3$.

bined with further work of Rudolph and Boileau-Orevkov, has the following consequence: Any knot realizable as the transverse intersection of an affine complex plane curve with a round three-sphere satisfies a generalized version of the slice-ribbon conjecture. That is, the minimal genus surface such a knot bounds in $B^{4}$ can be assumed to have no local maxima.

Unfortunately, none of the homology theories can be applied off the shelf in any obvious way to give more widely applicable information about the slice-ribbon conjecture. The great hope is to define a computable ribbon obstruction one might apply to a slice knot (for example, one of those constructed in a 2010 paper of Gompf, Scharlemann, and Thompson) to produce a counterexample to the conjecture. But the internal gradings in the theories cannot distinguish between a local maximum and a minimum of the radial function, so it is not clear how to proceed. There are hints, however, that so-called annular versions of the theories well suited to studying braids and their closures in the complement of the braid axis may have more success.

In my talk, I will focus on a link homology theory due to Khovanov and extended by E. S. Lee and J. Rasmussen (see Figure 3).

The annular version of this theory was defined by Asaeda-Przytycki-Sikora in 2004 and re-interpreted by L. Roberts in 2007. More recently, my collaborators-J. Baldwin, T. Licata, Y. Ni, and S. Wehrli-and I have made further

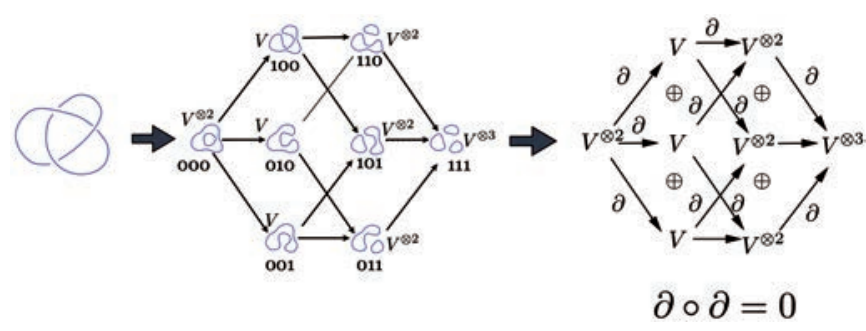

Figure 3. Khovanov discovered how to use the data of a link diagram to construct a multiply-graded chain complex whose homology is a link invariant. His construction was extended by Lee and Rasmussen to give strong information about surfaces in the fourball.

progress. While first attempts to find effective ribbon obstructions using this theory have been unsuccessful, we have uncovered a good deal of new information about braids, braid conjugacy classes, and braided surfaces. The annular version of the theory also carries additional algebraic structure, which has closely tracked the theory's topological and geometric applications. For example, it admits an action of the Lie algebra sl(2), and many key results and constructions can be phrased in terms of this action. With luck, this enhanced algebraic structure will point the way to other topological applications; or our failed attempts in this setting can be successfully carried out elsewhere; or both...

\section{Image Credits}

Figure 1 by Siddhi Krishna, @knotofthepress.https://drive. google.com/file/d/1IGmGXT1pRkXxDx0g2boedUaRyYZMg EnQ/view.

Figure 2 by Siddhi Krishna, @knotoffthepress.https://drive. google.com/fi1e/d/1xgQFmIoU1BCw7hnpu2EH54XTSR5LJ EJY/view.

Figure 3 and author photo courtesy of Elisenda Grigsby. 


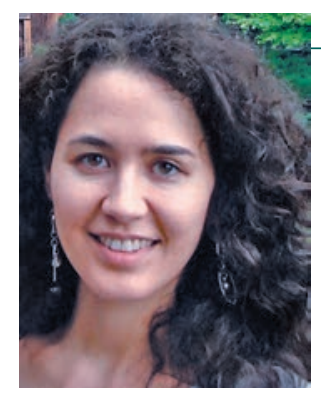

Elisenda Grigsby

\section{ABOUT THE AUTHOR}

Elisenda Grigsby's background is in low-dimensional topology. She has developed a side interest in the mathematics behind neural networks, which she blogs about as often as she can (that is, as often as her kids-ages 4 and 7-allow) at iamalearningcomputer. blogspot .com.
JOIN ALL
THREE

Members of MAA and SIAM, who are not currently

AMS members, are eligible to receive one year of AMS for $\$ 25$.

For more information about the JOIN3

promotion, please contact

Sales and Member Services at

cust-serv@ams.org or (800)321-4267.

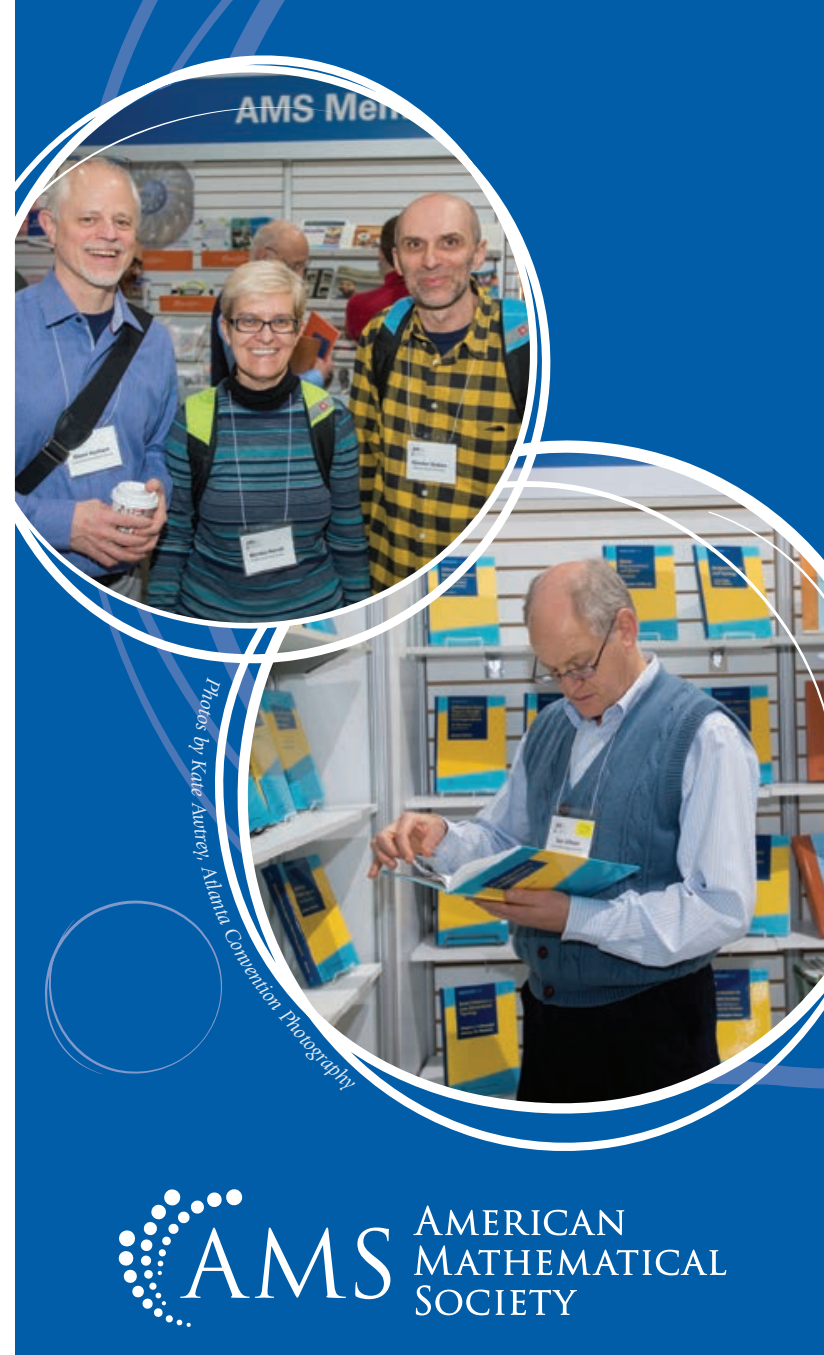

